\title{
Capillary introduction of polydimethylsiloxane precursor to microfabricated structure
}

\author{
Akihide Hibara ${ }^{1,2}$, Aoi Akiyama², Kotohiro Furukawa², Kyosuke Ishikawa², \\ ${ }^{1}$ Institute of Multidisciplinary Research for Advanced Materials, Tohoku University, 2-1-1 Katahira, \\ Aoba-ku, Sendai, Japan, \\ ${ }^{2}$ Department of Chemistry, Tokyo Institute of Technology, 2-12-1 Ookayama, Meguro-ku, Tokyo, \\ Japan \\ hibara@tohoku.ac.jp
}

\begin{abstract}
:
Capillary introduction colored polydimethylsiloxane (PDMS) precursor liquid to microfabricated straight channels was analyzed for opto-fluidic applications. The PDMS liquid was colored by Japanese black ink. The black PDMS is potentially used for light-shielding material after introduction. PDMS-glass microfluidic chips were used. Microchannels having in/out ports at both ends (open-end channel) and those having only one in/out port (dead-end channel) were prepared. The open-end channels were used for analyzing viscosity during the capillary introduction. Viscosity decrease with velocity was observed. Then, the dead-end channels were used for discussing the closed air effect on the capillary introduction. These informations will be useful for designing light-shielding in opto-microfluidic devices.
\end{abstract}

Key words: Capillarity, capillary introduction, opto-microfluidics, microfabrication, polydimethylsiloxane

\section{Introduction}

Micro/nanofabrication techniques have become one of essential technologies in modern chemical and bioanalytical devices. One of the most popular materials for the analytical devices is polydimethylsiloxane (PDMS) $[1,2]$. By placing objects on the mold before casting the precursor liquid, optical parts such as prisms can be embedded in a PDMS microfluidic device for opto-fluidic applications [3-5].

In order to control locus in integrated optical system, light-shielding parts should be placed to designed points. Here, we conceive a microfabricated light-shielding method, where black-colored PDMS precursor liquid is introduced to a patterned microchannel, and it will be light-shielding structure after solidification. One simple way to introduce PDMS liquid to a microchannel is utilization of capillary introduction. That kind of additional PDMS introduction to PDMS device has not been investigated.

In this study, we investigated PDMS liquid behaviors during its capillary introduction to a microchannel.

\section{Experimental}

Microfluidic devices were fabricated by ordinary soft-lithography method. Thick negative photoresist layer having a strait channel structure was used for a mold. The pattern was transferred to PDMS. Then, PDMS was bonded to a glass slide (straight microchannel device). The microchannel has a $100-\mu \mathrm{m}$ width, 34- $\mu \mathrm{m}$ height, and 30-mm length.

Black colored PDMS precursor (black PDMS) was prepared by adding (5\%) Japanese calligraphy black ink to ordinary PDMS precursor liquid.

Figure 1 illustrates the experimental procedure. A drop of black PDMS is put on an in/out port of the microfluidic device. PDMS liquid has high affinity to solidified PDMS and is spontaneously introduced to the channel. The introduction length of black PDMS is monitored on an optical microscope and recorded as a function of time. 


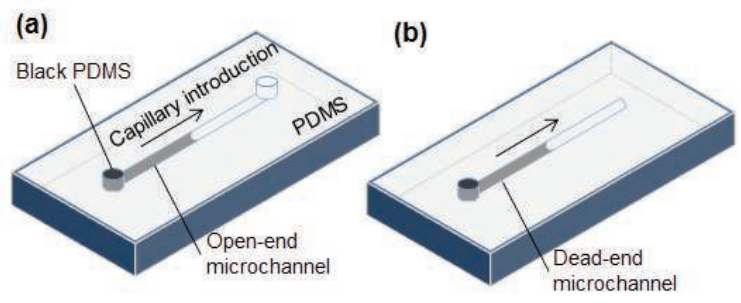

Fig. 1. Illustration of capillary introduction of PDMS precursor liquid to straight microchannels. (a) Openend channel. (b) Dead-end channel.

\section{Results and discussion}

Figure $2 a$ shows the result of capillary introduction length of black PDMS to the openend microchannel. By considering pressure loss by introduced liquid part, viscosity of black PDMS was calculated as Figure $2 \mathrm{~b}$. It has velocity dependence as $\mu_{\text {eff }} \propto \bar{v}^{-0.7}$.

(a)

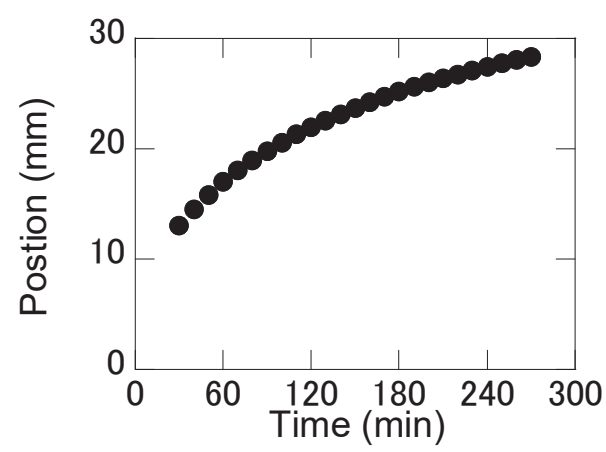

(b)

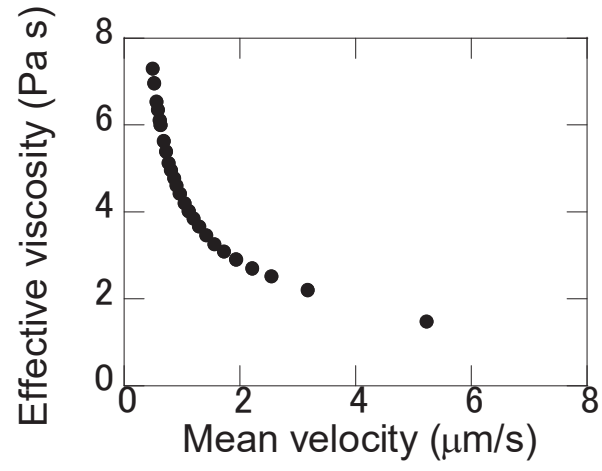

Fig. 2. (a) Introduction length of black PDMS to 30mm-long open-end channel. (b) Effective viscosity dependence on its speed.

Figure 3 shows the result of capillary introduction length of black PDMS to the deadend microchannel. The black PDMS was introduced to end of the channel. PDMS has air-permeability, and capillary force, which forced the liquid to the end direction, applied pressure to remaining air in the microchannel. Then air is removed through solidified PDMS.

The principle can be applied to fill lithographically designed (vacant) structure with black PDMS, which can act as light-shielding optical parts in opto-fluidic devices. We will show the applications of the light-shielding structures in the following studies.

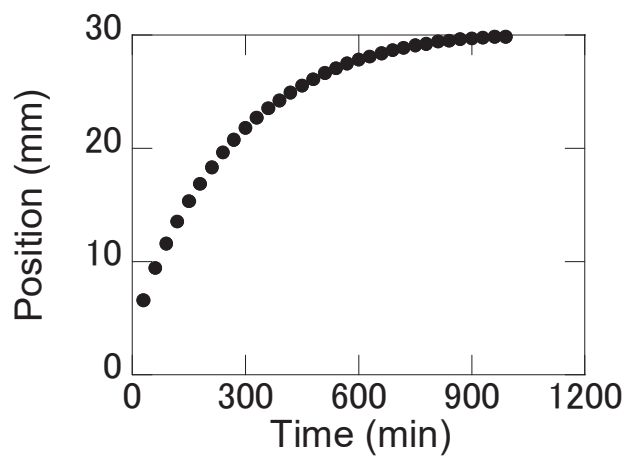

Fig. 3. Introduction length of black PDMS to 30 mm-long dead-end channel.

\section{References}

[1] JR Anderson, DT Chiu, RJ Jackman, O Cherniavskaya, JC McDonald, $\mathrm{H}$ Wu, SH Whitesides, and GM Whitesides, Analytical Chemistry, 72(14), 3158-3164 (2000). 10.1021/ac9912294

[2] M Fukuyama, M Tokeshi, MA Proskurnin, and A Hibara, Lab on a Chip, 18(2), 356-361 (2018). 10.1039/C7LC01121K

[3] Kazama and A Hibara, Analytical Sciences, 32(1), 99-102 (2016). 10.2116/analsci.32.99

[4] S Yamaoka, Y Kataoka, Y Kazama, Y Fujii, and A Hibara, Sensors and Actuators B: Chemical, 228, 581-586 (2016). 10.1016/j.snb.2016.01.072

[5] Y Kazama, ET Carlen, A van den Berg, and A Hibara, Sensors and Actuators B: Chemical, 248, 753-760 (2017). 10.1016/j.snb.2017.04.013 